(c) 2018 IEEE. Personal use of this material is permitted. Permission from IEEE must be obtained for all other uses, in any current or future media, including reprinting/republishing this material for advertising or promotional purposes, creating new collective works, for resale or redistribution to servers or lists, or reuse of any copyrighted component of this work in other works. 


\title{
Interactive Probabilistic Post-mining of User-preferred Spatial Co-location Patterns
}

\author{
Lizhen Wang", Xuguang Bao", Longbing Cao* \\ ${ }^{\text {\#} S c h o o l ~ o f ~ I n f o r m a t i o n ~ S c i e n c e ~ a n d ~ E n g i n e e r i n g, ~ Y u n n a n ~ U n i v e r s i t y, ~ K u n m i n g, ~ P . ~ R . ~ C h i n a ~}$ \\ *Advanced Analytics Institute, University of Technology Sydney, Sydney, Australia
}

\begin{abstract}
Spatial co-location pattern mining is an important task in spatial data mining. However, traditional mining frameworks often produce too many prevalent patterns of which only a small proportion may be truly interesting to end users. To satisfy user preferences, this work proposes an interactive probabilistic post-mining method to discover user-preferred colocation patterns from the early-round of mined results by iteratively involving user's feedback and probabilistically refining preferred patterns. We first introduce a framework of interactively post-mining preferred co-location patterns, which enables a user to effectively discover the co-location patterns tailored to his/her specific preference. A probabilistic model is further introduced to measure the user feedback-based subjective preferences on resultant co-location patterns. This measure is used to not only select sample co-location patterns in the iterative user feedback process but also rank the results. The experimental results on real and synthetic data sets demonstrate the effectiveness of our approach.
\end{abstract}

\section{INTRODUCTION}

The extraction of spatial co-location patterns is a rising and promising field in spatial data mining. A spatial co-location pattern is composed of a set of spatial features frequently observed together within geographical neighborhoods [1], [2]. Spatial co-location pattern mining yields important insights for various applications such as Earth science [3], public transportation [4], and air pollution [5].

Typically, spatial co-location pattern mining methods use the frequencies of a set of spatial features participating in a co-location pattern to measure a pattern's prevalence (known as participation index, $P I$ for short) and require a userspecified minimum prevalence threshold min_prev to filter prevalent co-location patterns [1], [2], [6]. However, User's preferences are often subjective, a pattern preferred by one user may not be favoured by another, thus cannot be measured by existing objective-oriented PI measures. Therefore, it is necessary and advantageous to involve user's preferences [7], [8], [9], [10], [11].

This work proposes a framework to discover user-preferred co-location patterns by iteratively involving user's interactive feedback and probabilistically quantifying user-preferences on co-location patterns. As shown in Fig. 1, our system takes a set $P C$ of mined prevalent co-locations as input. First, the top$k$ (e.g., $k=5$, in prevalence value order) co-locations in $P C$ are presented to the user as sample co-locations, and the system then asks the user for his/her preferences. The user chooses a set of preferred co-locations and so the first set $P C_{\text {feedback }}$ of selected co-locations is collected. Based on $P C_{\text {feedback }}$, the prevalent co-locations in $P C$ are estimated for their subjective preference by a probabilistic model, and ranked by their estimated subjective preferences. Then, as the sample colocations, the top- $k$ co-locations are fed to the user again. After several rounds of the interactive process, the system refines the output that is closest to the user's preference on colocation patterns.

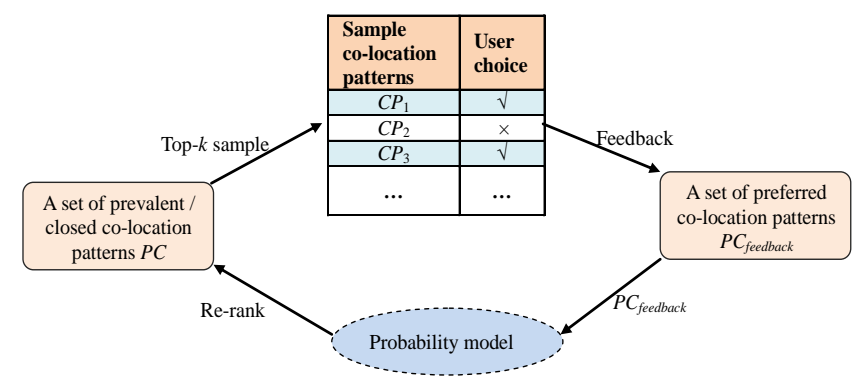

Fig. 1. A framework for interactively post-mining preferred co-locations

The rest of the paper is organized as follows. First, we review concepts related to traditional co-location mining, and formally defines our problem. Next, the probabilistic model method is proposed, and then presents our evaluation strategy and results.

\section{Problem Statement}

\section{A. Co-location Patterns}

In a spatial database, let $F$ be a set of $n$ features $F=\left\{f_{1}\right.$, $\left.f_{2}, \ldots, f_{n}\right\}, D$ be a set of instances of $F$, where each instance is a tuple <feature type, instance ID, location>, and $R$ be a neighbour relationship over locations of instances, where $R$ is symmetric and reflexive.

A co-location pattern $c$ is a subset of the feature set $F$. The number of features in $c$ is called the size of $c$. A row instance I of a co-location $c$ is a set of instances in $D$, which includes the instances of all features in $c$ and forms a clique under the neighbour relationship $R$. The set of all row instances of $c$ is called table instance of $c$, denoted as $T I(c)$.

The participation ratio of feature $f_{i}$ in a co-location $c$, denoted as $\operatorname{PR}\left(f_{i}, c\right)$, is the fraction of the instances of $f_{i}$ that participates in table instance $T I(c)$ of $c$. The participation index of a co-location $c$, denoted as $P I(c)$, is the minimum participation ratio $\operatorname{PR}\left(f_{i}, c\right)$ among all features $f_{i}$ in $c$. A colocation pattern $c$ is a prevalent co-location pattern, if its 
participation index PI is no less than a given prevalence threshold min_prev, that is, $P I(c) \geq$ min prev.

The PI and PR measures satisfy the anti-monotonicity property (downward closure property) [6]. The introduction of closed co-location patterns creates a lossless condensed representation. A prevalent co-location $c$ is closed if there is no co-location $c^{\prime}$ such that $c \subset c^{\prime}$ and $P I(c)=P I\left(c^{\prime}\right)[12]$.

\section{A. Subjective Preference Measure}

We assume that a set $P C$ of prevalent or closed co-location patterns has been mined which forms the input to our system. In $P C$, we suppose that there is a set $P C_{I}$ of ideal co-location patterns of interest to the user, and then $P C_{I}$ is the preferred co-location set and $P C_{I I}=P C-P C_{I}$ is the non-preferred colocation set.

We know that the value $P I(c)(c \in P C)$ is an objective interest measure in the prevalent co-location pattern mining. In reality, it is not possible that the objective interest can be substituted for subjective preference. $P C$ might contain a large number of mined prevalent co-location patterns, which may not be actionable and useful for users, since they may just be general knowledge, their prevalence may have been enhanced by the instances' autocorrelation, or they are just not preferred by the user.

In order to learn the prior knowledge of the user, we interactively ask for user's feedback concerning preferred colocations. User's feedback is combined into a set of preferred co-location patterns which is denoted $P C_{\text {feedback }}$. The set $P C_{\text {feedback }}$ is updated every time the system obtains user's feedback. Therefore, in the interactive process, we use a similarity measure $\operatorname{SIM}\left(c, P C_{\text {feedback }}\right)$ between a co-location pattern $c$ in $P C$ and the selected co-location patterns $P C_{\text {feedback }}$ to evaluate the degree of subjective preference of any colocation pattern $c$ which has not yet been judged.

\section{B. Problem Statement}

The problem of post-mining preferred spatial co-location patterns through interactive feedback can be stated as follows. Given a set of prevalent or closed co-location patterns, can the system return the ideal co-location patterns of user's preference, according to user's feedback about preferred colocations, and at the same time minimize the user's efforts in providing feedback?

Considering the uncertainties of the user's ideal preference, in this paper we use a classic probabilistic model to model the prior knowledge of the user. The basic idea of this method is: given a set $P C$ of prevalent or closed co-location patterns, there exists a set $P C_{I}$ of ideal preference co-location patterns in $P C$ for a user. However, the system does not know the characteristics of the set $P C_{I}$ at the beginning of the interactive process. It needs to make a guess. According to this guess, the system will identify a result set $P C_{I}$ as an initial hit. Then the user or system judges the initial result $P C_{I}$. Based on the feedback, the system can optimize and improve the initial result $P C_{I}$ incrementally in an interactive process so that, after repeated interactions, the resultant $P C_{I}$ should be close to the user's ideal preference result set.
The essence of the above probabilistic model is to estimate the probability of the similarity $\operatorname{SIM}\left(c, P C_{\text {feedback }}\right)$ between the selected co-locations $P C_{\text {feedback }}$ per user's feedback and a colocation pattern $c$ in the set of prevalent or closed co-locations $P C$ whose preference level has not yet been judged.

\section{PROBABILISTIC MODEL}

Assume, for a user, there is a preferred co-location set $P C_{I}$ and also a non-preferred co-location set $P C_{I I}$ in the prevalent or closed co-location set $P C$. After obtaining a set $P C_{\text {feedback }}$ of user's feedback, the similarity $\operatorname{SIM}\left(c, P C_{\text {feedback }}\right)$ between a colocation pattern $c$ in $P C$ and the set $P C_{\text {feedback }}$ per user's feedback is defined as the ratio of the probability of $c$ being of preference to the user compared to the probability of $c$ not being of preference to the user. i.e.,

$$
\operatorname{SIM}\left(c, P C_{\text {feedback }}\right)=\frac{P\left(P C_{I} \mid c\right)}{P\left(P C_{I I} \mid c\right)}
$$

where $P\left(P C_{I} \mid c\right)$ represents the probability of $c$ being of preference to the user, and $P\left(P C_{I I} \mid c\right)$ represents the probability of $c$ not being of preference to the user.

Since the values of $P\left(P C_{I} \mid c\right)$ and $P\left(P C_{I I} \mid c\right)$ cannot be computed directly, they need to be estimated with known values. Assume there is an initial guess about the user's ideal preference set $P C_{I}$, so Eqn. (1) can be converted per the Bayes' rule:

$$
\operatorname{SIM}\left(c, P C_{\text {feedback }}\right)=\frac{P\left(c \mid P C_{I}\right) \times P\left(P C_{I}\right)}{P\left(c \mid P C_{I I}\right) \times P\left(P C_{I I}\right)}
$$

where $P\left(c \mid P C_{I}\right)$ represents the probability that $c$ belongs to $P C_{I} ; P\left(c \mid P C_{I I}\right)$ represents the probability that $c$ belongs to $P C_{I I}$, and $P\left(P C_{I}\right)$ and $P\left(P C_{I I}\right)$ represent the prior probabilities that any co-location in $P C$ belongs to $P C_{I}$ or $P C_{I I}$ respectively.

For a given prevalent or closed co-location set $P C$, the two values $P\left(P C_{I}\right)$ and $P\left(P C_{I I}\right)$ are related only to the user but not to $c$. Additionally, we are just concerned about the relative values in computing SIM. So Eqn. (2) can be simplified to:

$$
\operatorname{SIM}\left(c, P C_{\text {feedback }}\right)=\frac{P\left(c \mid P C_{I}\right)}{P\left(c \mid P C_{I I}\right)}
$$

The probability of randomly selecting $c$ from $P C_{I}$ or $P C_{I I}$ (i.e., the probability that $c$ belongs to $P C_{I}$ or $P C_{I I}$ ) can be calculated by the distribution of each 2-size co-location $c_{i}$ in $P C_{I}$ and $P C_{I I}$ :

$$
\begin{aligned}
& P\left(c \mid P C_{I}\right)=\prod_{i=1}^{m} p\left(c_{i} \mid P C_{I}\right)^{w_{i}(c)} P\left(\bar{c}_{i} \mid P C_{I}\right)^{\left(1-w_{i}(c)\right)} \\
& P\left(c \mid P C_{I I}\right)=\prod_{i=1}^{m} p\left(c_{i} \mid P C_{I I}\right)^{w_{i}(c)} P\left(\bar{c}_{i} \mid P C_{I I}\right)^{\left(1-w_{i}(c)\right)}
\end{aligned}
$$

where $m=\frac{n(n-1)}{2}$, where $n$ is the number of features in $F$. $w_{i}(c) \in\{0,1\}: w_{i}(c)=1$ when the $i$-th 2-size co-location $c_{i}$ of $F$ is in $P C_{\text {feedback }}$ and $c$ at the same time; otherwise $w_{i}(c)=0$, and $\bar{c}_{i}$ represents "not containing 2-size co-location $c_{i}$."

Eqns. (4) and (5) can be interpreted as follows: when the 2size co-location $c_{i}$ is in $P C_{\text {feedback }}$ and $c$ at the same time, i.e., $w_{i}(c)=1$, the probability that $c_{i}$ appears randomly in a colocation pattern of $P C_{I}$ is regarded as a contribution to the process of determining whether $c$ and $P C_{I}$ are related. In the contrary situation, when the 2-size co-location $c_{i}$ is not the 
same as in $P C_{\text {feedback }}$ and $c$, the probability that $c_{i}$ does not appear randomly in a co-location pattern of $P C_{I}$ is also regarded as a contribution.

Based on Eqns. (4) and (5), Eqn. (3) can be converted to:

$$
\operatorname{SIM}\left(c, P C_{\text {feedback }}\right)=\frac{\prod_{i=1}^{m} p\left(c_{i} \mid P C_{I}\right)^{w_{i}(c)} P\left(\bar{c}_{i} \mid P C_{I}\right)^{\left(1-w_{i}(c)\right)}}{\prod_{i=1}^{m} p\left(c_{i} \mid P C_{I I}\right)^{w_{i}(c)} P\left(\bar{c}_{i} \mid P C_{I I}\right)^{\left(1-w_{i}(c)\right)}}
$$

Considering the meanings of $P\left(c_{i} \mid P C_{I}\right)$ and $P\left(\bar{c}_{i} \mid P C_{I}\right)$, we have $P\left(c_{i} \mid P C_{I}\right)+P\left(\bar{c}_{i} \mid P C_{I}\right)=1$. Accordingly, $P\left(c_{i} \mid P C_{I I}\right)+P\left(\bar{c}_{i} \mid P C_{I I}\right)=1$ holds. We take these relations into Eqn. (6) and by taking logarithms, it is converted to:

$$
\begin{aligned}
& \operatorname{SIM}\left(c, P C_{\text {feedback }}\right)= \\
& \sum_{i=1}^{m} w_{i}(c) \log _{10} \frac{p\left(c_{i} \mid P C_{I}\right)\left(1-P\left(c_{i} \mid P C_{I I}\right)\right.}{p\left(c_{i} \mid P C_{I I}\right)\left(1-P\left(c_{i} \mid P C_{I}\right)\right.}+ \\
& \sum_{i=1}^{m} \log _{10} \frac{1-P\left(c_{i} \mid P C_{I}\right)}{1-p\left(c_{i} \mid P C_{I I}\right)}
\end{aligned}
$$

As in Eqn. (7), the expression $\sum_{i=1}^{m} \log _{10} \frac{1-P\left(c_{i} \mid P C_{I}\right)}{1-p\left(c_{i} \mid P C_{I I}\right)}$ is not related to $c$, so Eqn. (7) can be further simplified as:

$$
\begin{aligned}
& \operatorname{SIM}\left(c, P C_{\text {feedback }}\right)= \\
& \qquad \sum_{i=1}^{m} w_{i}(c) \log _{10} \frac{p\left(c_{i} \mid P C_{I}\right)\left(1-P\left(c_{i} \mid P C_{I I}\right)\right.}{p\left(c_{i} \mid P C_{I I}\right)\left(1-P\left(c_{i} \mid P C_{I}\right)\right.}
\end{aligned}
$$

That is to say, we can compute the similarity of colocations $c$ with $P C_{\text {feedback }}$ by Eqn. (8), and rank them with the values $\operatorname{SIM}\left(c, P C_{\text {feedback }}\right)$. However, as mentioned before, the user's preference set $P C_{I}$ is not known initially. We need a method to calculate the probabilistic values $p\left(c_{i} \mid P C_{I}\right)$ and $p\left(c_{i}\right.$ | $\left.P C_{I I}\right)$.

A simple method for calculating the probabilistic values $p\left(c_{i} \mid P C_{I}\right)$ and $p\left(c_{i} \mid P C_{I I}\right)$ is that:

$$
\left\{\begin{array}{l}
p\left(c_{i} \mid P C_{I}\right)=0.5 \\
p\left(c_{i} \mid P C_{I I}\right)=n_{i} / N
\end{array}\right.
$$

where $n_{i}$ and $N$ represent the number of co-locations containing 2-size $c_{i}$ and the number of total co-locations in $P C$ respectively. We can then calculate the $\operatorname{SIM}\left(c, P C_{\text {feedback }}\right)$ for each $c$ in $P C$ by Eqn. (8).

However, Eqn. (9) is too arbitrary. After obtaining user's feedback information, based on the feedback principle we propose two improved methods (Eqn. (10) and Eqn. (11)) for calculating $p\left(c_{i} \mid P C_{I}\right)$ and $p\left(c_{i} \mid P C_{I I}\right)$ which improve the computation of $\operatorname{SIM}\left(c, P C_{\text {feedback }}\right)$ and help minimize the user's efforts in providing feedback.

$$
\left\{\begin{array}{l}
p\left(c_{i} \mid P C_{I}\right)=\left(r_{i}+0.5\right) /(r+1) \\
p\left(c_{i} \mid P C_{I I}\right)=\left(n_{i}-r_{i}+0.5\right) /(N-r+1)
\end{array}\right.
$$
(11).

Or, by adding less arbitrary adjusting factors, we have Eqn.

$$
\left\{\begin{array}{l}
p\left(c_{i} \mid P C_{I}\right)=\left(r_{i}+n_{i} / N\right) /(r+1) \\
p\left(c_{i} \mid P C_{I I}\right)=\left(n_{i}-r_{i}+n_{i} / N\right) /(N-r+1)
\end{array}\right.
$$

where $r$ is an adjusting factor (it can be pre-specified by the user) used to get a collection $V$ of the top- $r$ co-location patterns under the values $\operatorname{SIM}\left(c, P C_{\text {feedback }}\right)$, and $r_{i}$ is the number of co-location patterns containing 2-size co-location $c_{i}$ in $V$.

Note that Eqn. (9) needs to be used at the beginning of the interactive process when using Eqn. (10) or Eqn. (11).

\section{EXPERIMENTAL RESULTS}

We conduct comprehensive experiments to evaluate the proposed approach from multiple perspectives on both real and synthetic data sets. Due to space limitations, we present only a subset of our full results here.

\section{A. Experimental Setting}

We set up an experimental environment, called Simulator, to simulate user's feedback. Since our goal is to discover preferred co-location patterns interactively and rank the results, our accuracy measure favours high-rank co-location patterns in the results. Let top-l(learned_set) be the top-l results reported by the ranking learned from the interactive feedback and target_set be the results in the target colocations constructed by our Simulator, the accuracy measure is defined as follows.

$$
\text { Accurary }=\frac{\text { top }-l(\text { learned_set }) \cap \text { target_set }}{l}
$$

where $l$ is given $m / 5,2 m / 5,3 m / 5,4 m / 5$ or $m(m=\mid$ target_set $\mid)$ in the experiments. It is obvious that the accuracy values in Eqn. (12) are the percentages of the top- $l$ ranked co-locations in the target_set.

\section{B. Accuracy Evaluation on Real Data Sets}

Using the Simulator discussed above, our first task is to examine the accuracy of the results learned from the interactive feedback. We use three real data sets with different distributions in the experiments. Real-1 is from the rare plant data of the Three Parallel Rivers of Yunnan Protected Areas whose instances form a zonal distribution, which has a small quantity of instances. Real-2 is a spatial distribution data set of urban elements whose instances' distribution is both even and dense, and which has a large quantity of features as well as instances. Real-3 is a vegetation distribution data set of the Three Parallel Rivers of Yunnan Protected Areas, which has the fewest features but the most instances, and instance distribution presents various clusters.

We summarize the main lessons from the experiments.

First, over the three real data sets, we observed that F-10 and F-11 have better accuracy than F-9 (F-9, F-10 or F-11 means using Eqns. (9), (10) or (11) respectively to calculate the probabilistic values $p\left(c_{i} \mid P C_{I}\right)$ and $p\left(c_{i} \mid P C_{I I}\right)$ in calculating by Eqn. (8)) because F-10 and F-11 add some adjusting factors for computing $p\left(c_{i} \mid P C_{I}\right)$ and $p\left(c_{i} \mid P C_{I I}\right)$. The accuracy with F-11 is a little better than F-10 also because of the more reasonable probabilistic values. The accuracy estimated in closed co-locations is better than that in prevalent co-locations because closed co-locations are a form of compression of prevalent co-locations which can help effectively discover the interesting co-locations. We also find that: (1) as iter (number of iterations of feedback) increases, the accuracy increases, and this is because each iteration 
supplies new samples to the user, and the new feedback from the user updates the SIM values of co-locations in $P C$, bringing them closer and closer to the user's real preference; (2) a larger $k$ (number of sample co-locations for feeding to user) causes a higher accuracy because more samples can be fed to the user; (3) a smaller $l$ (number to get top-l(learned_set) for accuracy measure in experiments) can reach higher accuracy because the co-locations in the front of target_set have been already chosen by the user.

Second, the main observations on Real-2 are similar to Real-1, although the accuracy estimated for Real-2 is higher than that for Real-1 with the same parameter values, but the accuracy gap between prevalent co-locations and closed colocations is not as obvious as Real-1, because the compression of closed co-locations on Real-2 is much lower than that of Real-1, which makes a smaller gap between them.

Third, the accuracy on Real-3 can reach $100 \%$ within a few rounds. The reason for the high accuracy in Real-3 is that there are only 15 features, and the smaller number of features makes it easier to find the combinations preferred by a user.

\section{Accuracy Evaluation on Synthetic Data Sets}

Synthetic data sets are generated to test the accuracy and efficiency of our algorithm when data size changes. Figs. 2 and 3 show the accuracy and efficiency w.r.t. different number of features. We observe the following results.

First, the accuracy in a dense data set is higher than that in a sparse data set. The reason is that dense data sets can generate longer co-location patterns which have more chance of containing the preferred combination of features (rules), which means that preferred co-locations can be selected more easily in each round, further improving the accuracy of our algorithm.

Second, as the number of features increases, F-10 and F-11 show much better accuracy than F-9 in Fig. 2, and the gap of accuracy between F-10/F-11 and F-9 also increases. This is because the adjusted factors added in F-10 and F-11 play a bigger role as the data set gets bigger and bigger. Note that in this experiment there are about 1000000 spatial instances with 100 features.

Third, Fig. 3 shows the average running time of F-9, F-10 and F-11 per round and the number of closed co-locations (PC_count). It can be seen that F-9 has a much higher efficiency than either F-10 or F-11. When the number of closed co-locations reaches almost 700000, F-9 only costs no more than 20 seconds, and this is because F-9 only needs to calculate $n_{i}$, and $n_{i}$ can be updated based on the last round value. While F-10 and F-11 need to calculate not only $n_{i}$ but also $r_{i}$, and $r_{i}$ cannot be updated as $n_{i}$ because in each round the top- $r$ co-locations based on the SIM values may change greatly, thus increasing the running time. But even with 100 features, the running time per round is only around 70 seconds.

Acknowledgements This work was supported in part by grants (No. 61472346, No.61662086) from the National Natural Science Foundation of China, in part by a grant (No. 2016FA026, No.
2015FB114) from the Science Foundation of Yunnan Province, and in part by the Project of Innovation Research Team of Yunnan Province.

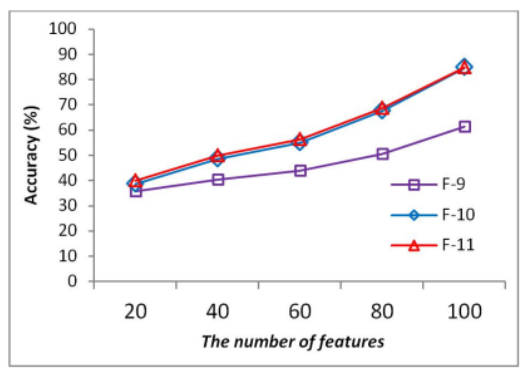

Fig. 2. Accuracy evaluation on synthetic data sets with different number of features

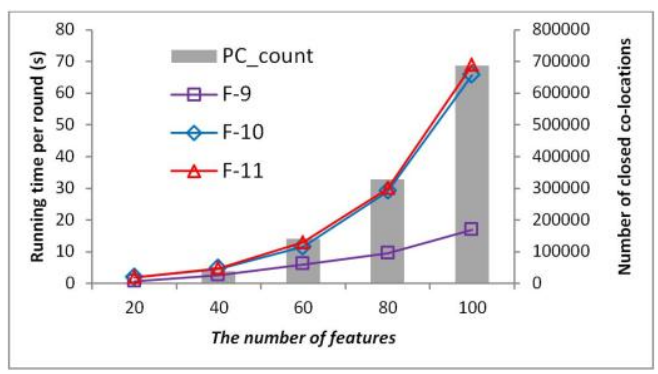

Fig. 3. Running time per round and the number of closed colocations with different number of features

\section{REFERENCES}

[1] S. Shekhar, and Y. Huang, "Co-location rules mining: a summary of results," in Proc. SSTD 2001, LNCS, vol. 2121, pp. 236-256.

[2] X. Zhang, N. Mamoulis, D. Cheung, and et al, "Fast mining of spatial co-locations," in Proc. ACM SIGKDD 2004, pp. 384-393.

[3] F. Verhein, G and Al-naymat, "Fast mining of complex spatial colocation patterns using GLIMIT," in: Proc. ICDMW 2007, pp. 679-684

[4] W. Yu, "Spatial co-location pattern mining for location-based services in road networks," Expert Systems with Applications, vol. 46, pp. 324 335, 2016.

[5] M. Akbari, F. Samadzadegan, and R. Weibel, "A generic regional spatio-temporal co-occurrence pattern mining model: a case study for air pollution," J. Geograph. Syst., vol. 17, pp. 249-274, 2015.

[6] Y. Huang, S. Shekhar, H. Xiong, "Discovering colocation patterns from spatial data sets: a general approach," IEEE Trans. Knowl. Data Eng (TKDE). vol. 16, no. 12, pp. 1472-1485, Dec. 2004.

[7] L. Wang, X. Bao, L. Zhou, "Redundancy reduction for prevalent colocation patterns," IEEE Trans. Knowl. Data Eng (TKDE), vol. 30, no. 1, pp. 142-155, Jan. 2018.

[8] L. Cao, Y. Zhao, H. Zhang, D. Luo, C. Zhang. "Flexible frameworks for actionable knowledge discovery," IEEE Trans. Knowl. Data Eng (TKDE), vol. 22, no. 9, pp, 1299-1312, Sep. 2010.

[9] D. Xin, X. Shen, Q. Mei, and J. Han, "Discovering interesting patterns through user's interactive feedback," in Proc. 12th ACM SIGKDD Int'l Conf. Knowledge Discovery and Data Mining, pp. 773-778, 2009.

[10] X. Bao, L. Wang, and H. Chen, "Ontology-based interactive postmining of interesting co-location patterns," in Proc. of the 18th AsiaPacific Web Conference (APWeb'16), LNCS vol. 9932, pp. 406-409, 2016.

[11] X. Bao, and L. Wang, "Discovering interesting co-location patterns interactively using ontologies," in DASFAA Workshops 2017, LNCS vol. 10179, pp. 75-89, 2017.

[12] J. S. Yoo and M. Bow, "Mining top-k closed co-location patterns," in Proc. IEEE Int'l Conf. Spatial Data Mining and Geographical Knowledge Services (ICSDM'11), pp. 100-105, 2011. 